\title{
Avaliação da Inserção do Estudante na Unidade Básica de Saúde: Visão do Usuário
}

\author{
Evaluation of Participation by Students in \\ Primary Healthcare Units: The User's View
}

Francisca Claudia Monteiro Almeida ${ }^{I}$ Ana Paula Pessoa Maciel ${ }^{I}$ Aline Ramos Bastos ${ }^{I I}$ Fernando Colares Barros ${ }^{I I}$ Juliana Ribeiro Ibiapinall Silézia Maria Franklin de Souzall Dayane Pessoa de Araújo II

\section{PALAVRAS-CHAVE: \\ - Avaliação em Saúde. \\ - Estudantes. \\ - Atenção Primária à Saúde.}

\section{KEYWORD:}

- Health Evaluation.

- Students.

- Primary Health Care.

Recebido em: 01/04/2010

Reencaminhado em: 30/08/2010

Aprovado em: 01/09/2010

REVISTA BRASLIEIRA DE EDUCAÇÃO MÉDICA 33 36 (1, Supl. 1): 33-39; 2012
${ }^{2}$ Secretaria Municipal de Saúde. Centro de Saúde da Família Edmar Fujita, Fortaleza, CE, Brasil; Universidade de Fortaleza, Fortaleza, CE, Brasil. "Universidade de Fortaleza, Fortaleza, CE, Brasil. 


\section{INTRODUÇÃO}

O Sistema Único de Saúde (SUS), modelo oficial público de atenção à saúde no Brasil, surgiu em um cenário de mudanças econômicas e políticas nos anos 1980, justificando a necessidade de um novo modelo que fosse diferente da assistência curativa e individual praticada à época. A criação do SUS ocorreu a partir de uma reforma sanitária, que teve seus princípios parcialmente incorporados pela Constituição Federal de $1988^{(1)}$.

A desarticulação entre as definições políticas dos Ministérios da Saúde e da Educação tem contribuído para acentuar o distanciamento entre a formação dos profissionais e as necessidades do SUS. Embora o SUS constitua um significativo mercado de trabalho para os profissionais de saúde, tanto nos serviços públicos quanto nos contratados, o seu impacto sobre o ensino de graduação na área de saúde ${ }^{(2)}$ tem sido pouco expressivo.

O cenário de trabalho no SUS e no mercado está mudando, e a Atenção Primária à Saúde (APS) passou a ser um espaço essencial para ajudar na formação de um profissional de saúde crítico, reflexivo, preparado para atuar em equipe. Os Ministérios da Saúde e da Educação, entendendo isso como uma necessidade para a adequada implantação do SUS, têm promovido várias iniciativas para estimular a aproximação das Universidades e dos serviços de saúde não hospitalares, tais como o Promed (Programa de Incentivo às Mudanças Curriculares das Escolas Médicas), o Pró-Saúde (Programa Nacional de Reorientação da Formação Profissional em Saúde), o PET-Saúde (Programa de Educação pelo Trabalho para a Saúde), etc. ${ }^{(3)}$.

A vivência de alunos de graduação na atenção básica condiz com um aprendizado diversificado, que não se limita apenas ao conhecimento teórico de condutas e procedimentos, mas se baseia fundamentalmente no relacionamento com os usuários inseridos em uma realidade própria, com necessidades e condições especiais.

A extensão universitária é um elemento fundamental na transformação do processo ensino-aprendizagem, atuando diretamente como elo entre a Universidade e a comunidade na construção de caminhos para a promoção social. A passagem pela extensão permite ao graduando a oportunidade de vivenciar a realidade de uma comunidade, observando de maneira crítica todos os aspectos que a influenciam ${ }^{(4)}$.

Os programas de extensão universitária desvelam a importância de sua existência na relação estabelecida entre instituição e sociedade, consolidando-se por meio da aproximação e troca de conhecimentos e experiências entre professores, alunos e população, pela possibilidade de desenvolvimento de processos de ensino-aprendizagem a partir de práticas cotidianas coadunadas com o ensino e a pesquisa e, especialmente, pelo fato de propiciar o confronto da teoria com o mundo real de necessidades e desejos. Na área da saúde, assumem particular importância na medida em que se integram à rede assistencial e podem servir de espaço diferenciado para novas experiências voltadas à humanização, ao cuidado e à qualificação da atenção à saúde ${ }^{(5)}$.

O estudo se justifica pela necessidade de avaliação da reorientação da formação profissional, sobretudo na área da saúde, visto que uma formação mais comprometida com a realidade contribui para a superação da dicotomia teoria-prática. Além disso, a educação com base na interdisciplinaridade certamente poderá levar à formação de profissionais mais comprometidos com a realidade de saúde e com a sua transformação.

A relevância do presente estudo se encontra na aplicação dos resultados obtidos em futuras pesquisas que sejam pertinentes aos temas em questão e, ainda, possibilita uma reflexão crítica entre os usuários dos serviços básicos de saúde acerca da importância da participação de estudantes de graduação na atenção primária.

Este estudo foi desenvolvido com o objetivo de avaliar sob o ponto de vista dos usuários a inserção do estudante de graduação na atenção básica.

\section{MÉTODOS}

Trata-se de um estudo exploratório-descritivo, com abordagem qualitativa e quantitativa. $\mathrm{O}$ trabalho foi realizado no Centro de Saúde da Família Dr. Edmar Fujita, que pertence à área de abrangência da Secretaria Executiva Regional VI (SER VI) do município de Fortaleza, no Ceará, e é integrante da rede de campos de estágio dos cursos de graduação do Centro de Ciências da Saúde da Universidade de Fortaleza - Unifor. Nele trabalham três equipes de Saúde da Família, um clínico geral e dois ginecologistas, oferecendo atividades diversas à população, tais como: consultas eletivas, pronto-atendimento em curativos, retirada de pontos, nebulizações e imunização. Além disso, conta com uma farmácia que é um polo de atendimento a usuários encaminhados de outras unidades de saúde da SER VI. Oferece ainda serviços de pré-natal, planejamento familiar, prevenção do câncer ginecológico e de mama, triagem neonatal, puericultura, acompanhamento ao hipertenso e ao diabético, controle da tuberculose e hanseníase, atendimento odontológico e vigilância epidemiológica.

A população do estudo constituiu-se de usuários do centro de saúde supracitado, sendo considerados para critérios de inclusão aqueles usuários que aceitaram participar da pesquisa e que aguardavam atendimento em consulta agendada. Inicialmente, 78 usuários foram abordados pelos discentes entrevistadores, mas apenas aqueles que tinham conhecimento da presença dos acadêmicos na unidade foram entrevistados. A 
amostra foi dimensionada por critérios de saturação do discurso, representando, ao final, 51 usuários. A saturação do discurso é um critério de representatividade da amostra para o encerramento da coleta de dados que permite abranger a totalidade do problema investigado em suas múltiplas dimensões ${ }^{(6)}$.

A coleta de dados foi realizada no período de dezembro de 2009 a fevereiro de 2010 por meio de escuta qualificada, auxiliada pela aplicação de entrevista estruturada aos participantes do estudo.

A etapa de análise dos dados abrangeu o tratamento estatístico das respostas às questões fechadas, com emprego do programa EPI-INFO, versão 3.5.1, e apresentação em quadros com os comentários pertinentes. As respostas às questões abertas foram analisadas quanto à frequência e à importância dada à justificativa dos sujeitos, sendo apresentadas seguindo a técnica de análise de conteúdo que diz ser “(...) um conjunto de técnicas de análise das comunicações que utiliza procedimentos sistemáticos e objetivos de descrição do conteúdo das mensagens" ${ }^{\prime \prime(7)}$.

A aceitação da participação dos sujeitos no estudo foi registrada em um Termo de Consentimento Livre e Esclarecido, de acordo com a Resolução 196/96(8) , garantindo-lhes o anonimato e o livre acesso para se desligarem da pesquisa. Os dados foram coletados após a emissão do parecer favorável do Comitê de Ética da Universidade de Fortaleza, sob nํㅜ 09.475.

\section{RESULTADOS E DISCUSSÃO}

Foram abordados 78 usuários dos serviços do Centro de Saúde da Família Dr. Edmar Fujita. Ao serem questionados sobre o conhecimento da presença dos acadêmicos na Unidade Básica de Saúde (UBS), 51 usuários $(65,4 \%)$ responderam que tinham conhecimento, enquanto outros $26(33,3 \%)$ responderam que não; e somente $1(1,3 \%)$ respondeu que conhecia parcialmente a presença dos estudantes. Os 51 usuários que referiram conhecer os acadêmicos foram entrevistados.

Em relação a esse questionamento inicial, destaca-se o percentual de usuários que desconhecem a presença dos acadêmicos. Isso pode decorrer do fato de eles não terem conseguido discriminar os profissionais da unidade dos discentes, devido à possível não identificação apropriada ou menção de sua condição por parte dos acadêmicos.

As respostas dos entrevistados às questões objetivas encontram-se sintetizadas no quadro 1 . Em relação às questões discursivas, pequena parte dos usuários não quis justificar a sua resposta. Alguns deles exibiram opiniões que se encaixavam em mais de uma categoria. As principais categorias temáticas identificadas no discurso dos usuários estão descritas no quadro 2.

A maioria dos 51 entrevistados $(90,2 \%)$ era do sexo feminino, totalizando 46 pessoas, enquanto $5(9,8 \%)$ eram do sexo masculino.

\section{QuAdro 1}

Questões objetivas relacionadas à inserção dos acadêmicos e frequências de respostas dos usuários entrevistados na

UBS $(\mathrm{n}=51)$

$\begin{array}{ll}\begin{array}{l}\text { Questões objetivas relacionadas à } \\ \text { inserção dos discentes na UBS Dr. } \\ \text { Edmar Fujita (n = 51) }\end{array} & \text { Frequências relativ } \\ \begin{array}{l}\text { 1. É importante a Unidade de Saúde } \\ \text { funcionar como campo de prática/ } \\ \text { atuação de acadêmicos universitários? }\end{array} & \begin{array}{l}\text { Não 1,95\% } \\ \text { Sim } 96,10 \%\end{array} \\ \begin{array}{l}\text { 2. Você acredita que os acadêmicos } \\ \text { universitários podem contribuir para }\end{array} & \text { Não 2,00\% } \\ \begin{array}{l}\text { a melhoria da qualidade da atenção à } \\ \text { saúde da comunidade? }\end{array} & \text { Sim } 98,00 \% \\ \begin{array}{l}\text { 3. O processo de aprendizagem dos } \\ \text { acadêmicos através da prática na }\end{array} & \text { Não 2,00\% } \\ \begin{array}{l}\text { Unidade de Saúde ajuda na formação do } \\ \text { futuro profissional? }\end{array} & \text { Sim 98,0\% } \\ \begin{array}{l}\text { 4. Concorda que a atuação dos acadêmicos } \\ \text { universitários de saúde da Unidade pode } \\ \text { desencadear mudanças em termos de } \\ \text { humanização da atenção à saúde? }\end{array} & \text { Sim 96,10\% }\end{array}$

5. Já assistiu alguma palestra/sessão de educação em saúde feita pelos acadêmicos na Unidade?

Desconhece 3,90\% Não $43,20 \%$ Sim $52,90 \%$

6. Concorda que são válidas/importantes Sim 100,00\% as atividades de educação em saúde realizadas pelos acadêmicos da área da saúde na Unidade?

7. Você já recebeu os acadêmicos universitários da Saúde em visita domiciliar?

8. Concorda que os acadêmicos universitários em estágio na Unidade valorizam os saberes que a comunidade já possui?

9. Já foi atendido aqui na Unidade de Saúde por um acadêmico?

10. Em que situação clínica foi o atendimento? (Responda caso tenha assinalado "sim" na questão 9; $\mathrm{n}=25$.)

11. Sente-se à vontade em ser assistido/ acompanhado por um acadêmico nos atendimentos da Unidade Básica de Saúde? (Responda caso tenha assinalado "sim" na questão 9; $\mathrm{n}=25$.)
Não $84,30 \%$

Sim $15,70 \%$

Não $5,90 \%$

Parcialmente 11,80\%

$\operatorname{Sim} 82,40 \%$

Não $51,00 \%$

Sim $49,00 \%$

Atendimento médico $52,17 \%$

Orientação

$21,7 \%$

Atendimento enfermagem $8,69 \%$

Parcialmente $4,00 \%$ $\operatorname{Sim} 96,00 \%$ 
Foi questionado aos usuários se eles consideravam importante a Unidade Básica de Saúde funcionar como campo de estágio para os alunos da graduação, fato que gerou uma resposta bastante positiva, pois $49(96,1 \%)$ deles consideraram importante, tendo como principais justificativas a oportunidade que os alunos têm de: "colocar o conhecimento em prática (...)"; "ajudar no atendimento do posto, melhorando (...)"; e "(..) aprenderem mais com a prática, com o contato com os pacientes, obtendo mais segurança", como mostra o quadro 2 . Somente 1 (1,95\%) entrevistado não considerou importante, alegando que "os alunos, algumas vezes, atrapalham o serviço, em vez de ajudar"; e 1 (1,95\%) usuário considerou parcialmente importante, “(...) depende do tipo de profissional que estiver acompanhando o aluno". Esses dados mostram a percepção dos usuários de que os estudantes utilizam o ambiente da Unidade não só como um estágio curricular obrigatório, mas como meio de praticar conhecimentos adquiridos na Universidade, de auxiliar no atendimento à saúde e também de agregar experiências e valores sociais à sua formação profissional. O estágio é um processo fundamental na formação do aluno estagiário, pois é a forma de fazer a transição de estudante para profissional. É um momento da formação em que o graduando pode vivenciar experiências, conhecendo melhor sua área de atuação ${ }^{(9)}$. Essa junção da teoria com a prática "possibilita ao graduando desenvolver a postura de pesquisador, despertar a observação, ter uma boa reflexão crítica, facilidade de reorganizar as ações para poder reorientar a prática quando necessário"'(10).

\section{QuAdro 2}

Dimensões da inserção dos acadêmicos na UBS e categorias de respostas dos usuários entrevistados $(n=51)$

$\begin{array}{|ll|}\begin{array}{l}\text { Dimensões da inserção dos } \\ \text { discentes na UBS Dr. Edmar } \\ \text { Fujita }\end{array} & \begin{array}{l}\text { Categorias de respostas dos } \\ \text { usuários }\end{array} \\ \begin{array}{l}\text { Importância da UBS como } \\ \text { espaço de prática/atuação de } \\ \text { acadêmicos universitários. }\end{array} & \begin{array}{l}\text { Oportunidade de prática pelos } \\ \text { discentes. } \\ \text { Auxílio no atendimento. } \\ \text { Geração de aprendizado e de } \\ \text { habilidades em atendimento. }\end{array} \\ \begin{array}{l}\text { Contribuição dos acadêmicos } \\ \text { para a melhoria da qualidade } \\ \text { da atenção à saúde da } \\ \text { comunidade. }\end{array} & \begin{array}{l}\text { Organização e agilidade no } \\ \text { atendimento. } \\ \text { Maior atenção e paciência com } \\ \text { os usuários. }\end{array} \\ & \begin{array}{l}\text { Transmissão de conhecimentos } \\ \text { para os pacientes. }\end{array}\end{array}$

\begin{tabular}{|c|c|}
\hline $\begin{array}{l}\text { Papel da aprendizagem } \\
\text { através da prática na UBS para } \\
\text { formação profissional. }\end{array}$ & $\begin{array}{l}\text { Ganho de experiência e } \\
\text { segurança no atendimento. } \\
\text { Contato com profissionais e } \\
\text { pacientes. }\end{array}$ \\
\hline $\begin{array}{l}\text { Humanização da atenção à } \\
\text { saúde pelas ações dos alunos. }\end{array}$ & $\begin{array}{l}\text { Atenção exemplar com os } \\
\text { usuários. } \\
\text { Possibilidade de atenção e } \\
\text { escuta de todos pacientes. } \\
\text { Convívio dos alunos com os } \\
\text { pacientes e a realidade da } \\
\text { comunidade. }\end{array}$ \\
\hline $\begin{array}{l}\text { Conforto com a assistência } \\
\text { e acompanhamento por } \\
\text { acadêmicos na UBS. }\end{array}$ & $\begin{array}{l}\text { Atendimento bom. } \\
\text { Importância para o aprendizado } \\
\text { dos discentes. } \\
\text { Melhor qualidade e assistência } \\
\text { no atendimento. }\end{array}$ \\
\hline $\begin{array}{l}\text { Avaliação de palestra/sessão de } \\
\text { educação em saúde feita pelos } \\
\text { acadêmicos na Unidade. }\end{array}$ & $\begin{array}{l}\text { Atividade boa. } \\
\text { Informações bem transmitidas. } \\
\text { Esclarecimento de dúvidas e } \\
\text { aprendizado. }\end{array}$ \\
\hline $\begin{array}{l}\text { Validade das atividades de } \\
\text { educação em saúde pelos } \\
\text { acadêmicos na UBS. }\end{array}$ & $\begin{array}{l}\text { Esclarecimento de dúvidas e } \\
\text { reforço de orientações. } \\
\text { Forma de capacitação e } \\
\text { aprendizado para os alunos. } \\
\text { Maior compreensão dos usuários } \\
\text { sobre cuidados em saúde. }\end{array}$ \\
\hline $\begin{array}{l}\text { Atuação acadêmica em visita } \\
\text { domiciliar. }\end{array}$ & $\begin{array}{l}\text { Atuação importante e útil. } \\
\text { Boa dedicação e afeto dos } \\
\text { alunos. } \\
\text { Esclarecimento de dúvidas e } \\
\text { transmissão de orientações. }\end{array}$ \\
\hline $\begin{array}{l}\text { Valorização dos saberes da } \\
\text { comunidade pelos acadêmicos. }\end{array}$ & $\begin{array}{l}\text { Diálogo e escuta atenta pelos } \\
\text { alunos. } \\
\text { Interesse pelo saber da } \\
\text { comunidade e por práticas de } \\
\text { saúde locais. } \\
\text { Respeito e aceitação dos saberes } \\
\text { da comunidade. }\end{array}$ \\
\hline
\end{tabular}

Noventa e oito por cento (50) considera que a atuação do acadêmico contribui para a melhoria da qualidade da atenção à saúde na Unidade, na medida em que “(...) organizam o atendimento"; “(...) auxiliam no atendimento, na falta de profissionais"; "interagem bem com a população, ajudando, esclarecendo dúvidas (...)"; “(...) repassam, ensinam bem novos conhecimentos para a população e para os profissionais". Outros comentários dos usuários estão descritos no quadro 2. Esses dados sugerem a boa aceitação dos acadêmicos por parte dos usuários e mostram suas opiniões e perspectivas de que o trabalho dos alunos auxilia na melhoria da qualidade do serviço prestado na Unidade Básica de Saúde. A percepção da realidade das pessoas, das suas condições de vida, cultura e costumes permitem ao estudante construir uma concepção 
do processo saúde-doença na qual compreendem os determinantes e as relações das doenças com o modo de vida e trabalho das pessoas. Essa concepção possibilita uma mudança no cuidado à saúde dos usuários, família e comunidade, a qual permite um cuidado mais voltado para as ações de vigilância à saúde, o que leva à integralidade no cuidado do paciente, aprimorando, assim, as ações de atenção à saúde $\mathrm{e}^{(11)}$.

Ao serem questionados se o aprendizado por meio da prática ajuda na formação do futuro profissional dos estudantes, $98,0 \%$ dos usuários (50) responderam que sim, enquanto $1(1,95 \%)$ considerou que ajuda parcialmente. Essa visão dos usuários vem afirmar que a atuação do acadêmico na atenção básica proporciona-lhe uma visão ampliada da saúde, descartando a visão exclusivamente biológica transmitida pela Universidade, fazendo com que ele, o discente, passe a perceber o homem inserido em um contexto político, econômico e social. Sendo assim, a extensão possibilita a formação de um profissional da saúde mais comprometido com a realidade social, enfatizando a prevenção e a promoção da saúde ${ }^{(4)}$.

Com relação à humanização da atenção à saúde, 49 $(96,1 \%)$ pacientes da Unidade responderam que as ações dos discentes são favoráveis a esse processo, e 2 (3,90\%) responderam que as ações são parcialmente favoráveis.

Da análise dos discursos dos usuários, evidencia-se que alguns deles percebem grande dedicação, atenção no atendimento pelos discentes, chegando a afirmar que "os alunos podem ser modelos para os profissionais de como dar mais atenção". Já outros consideram que a maior humanização da atenção ocorre pelo fato de haver mais pessoas para atenderem os pacientes, o que permite que estes possam se expressar melhor e ser escutados.

Humanizar a atenção e a gestão em saúde se apresenta como meio para a qualificação das práticas de saúde: acesso com acolhimento; atenção integral e equânime com responsabilização e vínculo; valorização dos trabalhadores e usuários com avanço na democratização da gestão e no controle social participativo $^{(12)}$. Apesar da gama de entendimentos do constructo "humanização" pelos usuários, tanto do ponto de vista conceitual como de política pública de saúde, os discursos dos usuários pesquisados, por se referirem a um melhor acolhimento e acesso aos serviços de saúde, sugerem que as ações acadêmicas permitem uma maior humanização da atenção à saúde.

Dos 51 usuários entrevistados, 49\% (25) foi atendido ou acompanhado por algum estudante na Unidade, sendo que $52,7 \%$ (11) foi consultado por discente de Medicina, 8,69\% (2) por discente de Enfermagem, sempre acompanhado por um profissional da Unidade. Já os outros 51\% (26) não teve contato com os acadêmicos.
Com relação à segurança no atendimento dos estudantes, $24(96,0 \%)$ dos 25 pacientes atendidos ou acompanhados responderam que se sentiam confortáveis com a presença e o serviço prestados pelos discentes, e 1 (4,00\%) respondeu que não sentia o mesmo. Da análise dos discursos dos pacientes, pode-se perceber que a maioria dos entrevistados sente-se à vontade com o atendimento dos estudantes, acreditando que este seja equiparável ou até melhor do que o atendimento de um profissional: "(...) o atendimento é melhor, eles dão mais assistência, mais carinho aos pacientes". A boa relação acadêmico-usuário é de extrema importância para o desenvolvimento de ações em conjunto com a comunidade, visto que são os usuários os principais beneficiados com a atuação dos estudantes.

O estudo realizado ainda mostrou a percepção de 20 estudantes de Medicina da $1^{\text {a }}$ e $2^{\text {a }}$ séries da Faculdade de Medicina de Marília (Famema) em relação às atividades desenvolvidas na Atenção Básica de Saúde (ABS). Os discursos dos sujeitos evidenciam o papel social que eles têm nas relações com a comunidade, formando um elo entre o estudante, o serviço de saúde e a população na Atenção Básica em Saúde. Essa relação contribui para a melhoria das condições de saúde das pessoas, ao mesmo tempo em que colabora para a aprendizagem do estudante ${ }^{(11)}$.

Ao serem questionados sobre o acompanhamento prévio de alguma atividade de educação em saúde (palestra, oficina), $52,90 \%$ (27) dos usuários entrevistados referiram ter acompanhado alguma atividade, enquanto 47,10\% respondeu nunca ter acompanhado ou não soube informar. Para a maioria dos que afirmaram ter assistido alguma atividade ministrada por acadêmicos, a atividade foi satisfatória, pois as dúvidas, segundo eles, foram sendo esclarecidas e os alunos "ensinaram bem as informações, com calma, atenção, dedicação".

Já em relação à importância das atividades de educação em saúde, os 51 (100\%) pacientes concordaram que elas são válidas. Da análise dos discursos, verificou-se que a maioria dos usuários percebe as atividades como uma forma de obter mais informações sobre saúde, de aumentar a sua compreensão sobre as doenças e os cuidados em saúde, chegando alguns, inclusive, a afirmar que as atividades "ajudam a melhorar a saúde".

Assim, o discurso dos usuários reforça a ideia de que a educação em saúde constitui um recurso por meio do qual o conhecimento cientificamente produzido no campo da saúde, intermediado pelos profissionais dessa área, atinge a vida cotidiana das pessoas, uma vez que a compreensão dos condicionantes do processo saúde-doença oferece subsídios para a adoção de novos hábitos e condutas de saúde ${ }^{(13)}$. Já outros usuários destacaram que as atividades educativas na UBS são 
importantes para os alunos, pois estes "aprendem", “(...) capacitam-se para esclarecer dúvidas da população". Isso embasa a visão de que os estágios nas UBSs contribuem para a aprendizagem e formação profissional dos discentes, constituindo-se como oportunidade para o aprimoramento e extensão dos conhecimentos adquiridos no âmbito da faculdade.

Em relação às visitas domiciliares, 84,3\% (43) dos usuários disseram não ter recebido visita com a participação de alunos. Já entre aqueles que foram visitados por discentes (15,7\%), a maioria refere que a atuação destes "(...) foi boa, ótima”, "(...) foi importante e ajudou" e que os alunos "foram dedicados, atenciosos, afetuosos (...)”, “(...) esclareceram dúvidas".

A assistência domiciliar não modifica apenas a maneira de trabalhar, somando mais uma tarefa aos serviços de saúde tão saturados, mas tem potencialidades para sensibilizar o modo de agir e pensar dos profissionais. A prática de prestar assistência nos domicílios, nos lares, nos locais de vivência e trabalho das pessoas favorece uma aproximação da realidade que é complexa e dinâmica, possibilitando, portanto, uma reflexão e revisão da própria atitude dos profissionais na busca de transformações do cuidado ${ }^{(14)}$. De acordo com o discurso dos usuários, apesar das dificuldades de ordem operacional e social que as visitas domiciliares representam para os profissionais e os acadêmicos, uma maior regularidade e uma frequência de visitas dos discentes seriam bem-vistas e apoiadas pela população.

Segundo a opinião dos 51 usuários entrevistados, 82,4\% (42) considera que os acadêmicos valorizam os saberes próprios da comunidade, uma vez que os alunos “(...) exploram o saber da comunidade", “(...) interessam-se por práticas de saúde próprias da comunidade", “(...) respeitam, aceitam os saberes da comunidade" $\mathrm{e}$ "(...) conversam bastante com os pacientes, escutando sua opinião (...)".

A partir do diálogo e intercâmbio de saberes técnico-científicos e populares, profissionais e usuários podem construir de forma compartilhada um saber sobre o processo saúde-doença. Esse compromisso e vinculação com os usuários possibilita o fortalecimento da confiança nos serviços ${ }^{(13)}$. As opiniões dos usuários indicam que a maioria dos discentes reconhece a importância da valorização dos saberes da comunidade, já que, por meio do diálogo e da troca de conhecimentos, favorece-se o reconhecimento dos usuários enquanto sujeitos portadores de saberes sobre o processo saúde-doença-cuidado e de condições concretas de vida.

\section{CONCLUSÃO}

Neste trabalho, constatou-se a contribuição positiva da inserção do aluno de graduação nas Unidades Básicas de Saúde na opinião dos próprios usuários do serviço, visto que, com essa inserção comunitária, os estudantes tornam-se conhecedores e potenciais modificadores da realidade. Além disso, o julgamento favorável dos usuários em relação à presença e colaboração dos alunos em atividades voltadas à humanização, ao cuidado e à qualificação da atenção à saúde torna-se um subsídio para a formação de profissionais críticos, com consciência da realidade social na qual estão inseridos e com capacidade para desenvolver ações voltadas à prevenção, promoção e educação em saúde, ou seja, profissionais socialmente referenciados e comprometidos.

\section{AGRADECIMENTOS}

À professora Silézia Maria Franklin de Souza, tutora da "Árvore 1" do PET-Saúde/Unifor; aos profissionais da Unidade Básica de Saúde onde foi realizada a pesquisa; à Universidade de Fortaleza e ao Programa de Educação pelo Trabalho para a Saúde (PET-Saúde/Unifor).

Apoio: Fonte financiadora da pesquisa: PET-Saúde/Unifor.

\section{REFERÊNCIAS}

1. Brasil. Ministério da Saúde. Secretaria Executiva. Sistema Único de Saúde (SUS): princípios e conquistas. Brasília: Ministério da Saúde; 2000.

2. Campos FE, Ferreira JR, Feuerwerker L, Sena RR, Campos JJB, Cordeiro H, Cordoni Júnior L. Caminhos para aproximar a formação de profissionais de saúde das necessidades da atenção básica. Rev. Bras. Educ. Méd. 2001 mai-ago; 25 (2): 53-59.

3. Pinto MEB, Gama CM, Gonçalves MR, Souza AC de. Experiência interdisciplinar em equipe multiprofissional na graduação na atenção primaria à saúde - Pet-Saúde UFCSPA.

4. Falcão EF. Vivência em comunidades: outra forma de ensino. João Pessoa: Editora Universitária; 2006.

5. Hennington EA. Acolhimento como prática interdisciplinar num programa de extensão universitária. Cad. Saúde Pública. 2005 jan-fev; 21 (1): 256-265.

6. Zoboli ELCP, Fortes PAC. Bioética e atenção básica: um perfil dos problemas éticos vividos por enfermeiros e médicos do Programa Saúde da Família, São Paulo, Brasil. Cad. Saúde Pública. 2004 nov-dez; 20(6):1690-1699.

7. Bardin L. Análise de conteúdo. Trad. Luiz Antero Reto e Augusto Pinheiro. Lisboa: Edições 70; 2002.

8. Ministério da Saúde (BR). Diretrizes e normas regulamentadoras de pesquisa envolvendo seres humanos (Resolução 196/96). Brasília: Ministério da Saúde; 1996.

9. Francisco CM, Pereira AS. Supervisão e sucesso do desempenho do aluno no estágio. [on-line]. [citado 2010 Mar 
13]. Disponível em: URL: http://www.efdeportes.com/ efd69/aluno.htm

10. Souza JCA, Bonela LA, De Paula AHA. Importância do estágio supervisionado na formação do profissional de educação física: uma visão docente e discente. MOVIMENTUM - Revista Digital de Educação Física. 2007. [on-line]. [citado 2010 Mar 13]. Disponível em: URL: http:/ /www. unilestemg.br/movimentum/index_arquivos/movimentum_V2_N2_souza_janua_luciane_bonela_2_2006.pdf

11. Ferreira RC, Silva RF, Aguer CB. Formação do profissional médico: a aprendizagem na atenção básica de saúde. Revista Brasileira de Educação Médica. 2007; 31 (1): 52-59.

12. Benevides R, Passos E. Humanización en la salud: ¿Un nuevo modismo?. Interface (Botucatu). 2005 Ago; 9 (17): 389-394.

13. Alves VS. Um modelo de educação em saúde para o Programa Saúde da Família: pela integralidade da atenção e reorientação do modelo assistencial. Interface (Botucatu). 2005 Fev; 9 (16): 39-52. [on-line]. [citado 2010 Mar 14]. Disponível em: URL: http://www.scielo.br/scielo.php?script=sci_ arttext\&pid=S1414-32832005000100004\&lng=en\&nrm=iso

14. Sakata KN. Concepciones del equipo de salud de la familia sobre das visitas domiciliarias. Rev. Bras. Enferm. 2007 Dez; 60 (6): 659-664. [on-line]. [citado 2010 Mar 14]. Disponível em: URL: http:/ / www.scielo.br/scielo.php?script=sci_art text\&pid $=$ S00341672007000600008\&lng=en\&nrm=iso

\section{CONTRIBUIÇÃO DOS AUTORES}

Silézia Maria Franklin de Souza participou na concepção e desenho do estudo. Francisca Cláudia Monteiro Almeida participou da coleta, revisão literária, análise, interpretação dos dados e da redação do texto. Aline Ramos Bastos e Dayane Pessoa de Araújo participaram da coleta, revisão literária e redação do texto. Fernando Colares Barros e Juliana Ribeiro Ibiapina participaram da coleta, análise, interpretação dos dados e da redação do texto. Ana Paula Pessoa Maciel participou da coleta e redação do texto.

\section{CONFLITO DE INTERESSES}

Declarou não haver.

\section{ENDEREÇO PARA CORRESPONDÊNCIA}

Francisca Cláudia Monteiro Almeida

Av. Alberto Craveiro, 1480

Boa Vista - Fortaleza

CEP. 60861-212 CE

E-mail: claudmonteiro@yahoo.com.br 